\title{
How Should the Distant Future be Discounted When Discount Rates are Uncertain?
}

\author{
Christian Gollier and Martin L. Weitzman*
}

November 7, 2009.

\begin{abstract}
It is not immediately clear how to discount distant-future events, like climate change, when the distant-future discount rate itself is uncertain. The so-called "WeitzmanGollier puzzle" is the fact that two seemingly symmetric and equally plausible ways of dealing with uncertain future discount rates appear to give diametrically opposed results with the opposite policy implications. We explain how the "Weitzman-Gollier puzzle" is resolved. When agents optimize their consumption plans and probabilities are adjusted for risk, the two approaches are identical. What we would wish a reader to take away from this paper is the bottom-line message that the appropriate long run discount rate declines over time toward its lowest possible value.
\end{abstract}

\section{Introduction}

The concept of discounting is central to economics, since it allows effects occurring at different future times to be compared by converting each future dollar into a common currency of equivalent present dollars. Because of this centrality, the choice of an appropriate discount rate is one of the most critical issues in economics. It is an especially acute issue for projects involving long time horizons because in such situations the results of cost-benefit analysis (CBA) can be incredibly sensitive to even tiny changes in the discount rate.

The primary motivator for this paper is the economics of climate change. Answers to questions thrown up by the climate-change CBA hinge critically on the core issue of how

\footnotetext{
*Gollier: Toulouse School of Economics (gollier@cict.fr); Weitzman: Department of Economics, Harvard University (mweitzman@harvard.edu). The research leading to these results has received funding from the European Research Council under the European Community's Seventh Framework Programme (FP7/20072013) Grant Agreement no. 230589.
} 
to discount the distant future. There is a high degree of uncertainty about what should be taken as the appropriate rate of return on capital in the long run, accompanied by much controversy about its implications for long-run discounting. The investigation of this paper is focused sharply on the well defined sub-problem of how to perform CBA of small incremental investments that might alter distant-future events, although the overall ramifications of the paper are somewhat broader.

The effects of global warming and climate change will be spread out over what might be called the "distant future" - centuries (or even millennia) from now. The logic of compounding a constant positive interest rate forces us to say that what we might conceptualize as monumental - even earth-shaking - events do not much matter when they occur in the distant future. Perhaps yet more disconcerting, when exponential discounting is extended over very long time periods there is a truly extraordinary dependence of CBA on the choice of a discount rate. Seemingly insignificant differences in discount rates can make an enormous difference in the present discounted value of distant-future payoffs. In many long-run situations, including climate change, almost any answer to a CBA question can be defended by one choice or another of a discount rate.

We think it is important to begin by recognizing that there is no deep reason of principle that allows us to extrapolate past rates of return on capital into the distant future. The seeming trendlessness of some past rates of return is a purely empirical reduced-form observation, which is not based on any underlying theory that would confidently allow projecting the past far into the future. There are a great many fundamental non-extrapolatable factors, just one example of which is the unknown future rate of technological progress. Even leaving aside the question of how to project future interest rates, additional issues for climate change involve which interest rate to choose out of a multitude of different average rates of return that are out there in the past and present real world. Furthermore, there is an ethical dimension to discounting climate change across many future generations that is difficult to evaluate and incorporate into standard CBA. A very large number of additional examples of economic and non-economic features could be given that are currently unknown but would be highly relevant to determining the distant-future discount rate. The fundamental point is that there is enormous uncertainty and controversy about choosing an appropriate rate of return for discounting distant-future events, like climate change. Moreover, the great uncertainty about discounting the distant future is not just an academic curiosity, but it has critically important implications for climate change policy. This disturbing ambiguity has given rise to a great deal of controversy and a variety of proposed solutions. Our purpose here is to focus sharply on clarifying this particularly thorny issue by using a crisp formulation that abstracts away from all other elements of CBA. 
In this paper we construct the simplest possible model for analyzing how to discount the distant future for a CBA decision that must be made now, when future discount rates are uncertain. We do not defend this model for its realism and immediate applicability to such long-term issues as CBA of climate change. Rather, we defend our abstract optimizing model for its ability to isolate, clarify, and hopefully bring some closure to a set of controversial issues that have bedeviled the discounting of distant-future events like climate change.

The bottom line message we want a reader to take away from this paper: there exists a rigorous generic argument that the future should be discounted at a declining rate that approaches asymptotically its lowest possible value.

\section{Background: the "Weitzman-Gollier Puzzle"}

In this section we focus on a paradoxical issue, whose proposed resolution has featured prominently in a number of papers on discounting the distant future. We do not mean to imply that this is the only important issue in long-term discounting, or that our own resolution of the puzzle is the final word. We are primarily using this alleged paradox as a way of grabbing a reader's attention in order to motivate the rigorous analysis that comes with the next section of the paper.

In the highly stylized model of this paper, time $t=0,1,2, \ldots$, is measured in discrete periods of unit length. To state loosely the issue at hand, a decision must be taken now, just before time zero (call it time $0^{-}$), whether or not to invest a marginal cost $\delta$ that will yield a marginal benefit $\epsilon$ at future time $t$. Right now, at time $0^{-}$, it is unknown what will be the appropriate future rate of return on capital in the economy. There are $n$ possible future states of the economy, indexed by $i=1,2, \ldots, n$. As of now (time $0^{-}$), future state $i$ is viewed as having marginal product of capital $r_{i}$ with probability $p_{i}>0$, where $\Sigma_{i} p_{i}=1$. A decision must be made now (at time $0^{-}$, just before the "true" state of the world is revealed at time $t=0$ ) about whether or not to invest $\delta$ now in order to gain payoff $\epsilon$ at future time $t$. To pose the problem sharply, it is assumed that immediately after the investment decision is made, at time 0 , the true state of the world $i$ is revealed and the marginal product of capital will thenceforth be $r_{i}$, from time $t=0$ to time $t=\infty$. The idea that productivity shocks are permanent seems an appropriate abstraction for analyzing the distant-future discount rate. Even if we thought of interest rates as being a mean-reverting random variable, our best statistical estimate of this mean is itself a random variable, which makes the reduced-form overall stochastic process be non-mean-reverting.

In a pair of articles, Weitzman ${ }^{1}$ proposed the idea that what should be probability-

\footnotetext{
${ }^{1}$ See Weitzman (1998) and Weitzman (2002).
} 
averaged at various times is not future discount rates, but future discount factors. In other words, one should not apply the average discount rate $\Sigma_{i} p_{i} r_{i}$ as if it were a timeindependent constant. Instead one should apply the time-dependent average discount factor $A(t)=\Sigma_{i} p_{i} \exp \left(-r_{i} t\right)$, whose corresponding time-dependent "effective" discount rate $R^{W}(t)$ satisfies

$$
\exp \left(-R^{W}(t) t\right)=\sum_{i=1}^{n} p_{i} \exp \left(-r_{i} t\right)
$$

which can be rewritten as

$$
R^{W}(t)=-\frac{1}{t} \ln \left(\sum_{i=1}^{n} p_{i} \exp \left(-r_{i} t\right)\right)
$$

Accepting the above logic, it follows that the investment should be made (incurring a marginal $\operatorname{cost} \delta$ now, at time $0^{-}$, in order to yield a marginal benefit $\epsilon$ at future time $t$ ) if and only if

$$
\epsilon \exp \left(-R^{W}(t) t\right) \geq \delta
$$

It is not difficult to show that $R^{W}(t)$ defined by (2) has the properties

$$
R^{W}(0)=\sum_{i=1}^{n} p_{i} r_{i}, \frac{d R^{W}(t)}{d t}<0, \quad R^{W}(\infty)=\min \left\{r_{i}\right\}
$$

Unfortunately, Weitzman did not provide a rigorous story to accompany the idea that what should be probability-averaged at various times is not discount rates, but discount factors. Instead the argument in his papers was left at the intuitive or heuristic level. A main purpose of this paper is to provide a rigorous justification for an appropriate version of (1)-(4). A simple intuition is derived from observing that one can anticipate the future benefit $\varepsilon$ by reducing productive capital by the present value $\varepsilon \exp \left(-r_{i} t\right)$ at date 0 . This reduction will be fully compensated at date $t$ by the cash flow $\varepsilon$ generated by the project, so that all cash flows are concentrated at date 0 . In expectation, this net present value is positive if and only if condition (3) is satisfied.

In a series of articles, Gollier ${ }^{2}$ inverted Weitzman's logic to produce a seemingly symmetric discount-rate story with exactly the opposite properties. Gollier reasoned along the following lines. One can transfer the initial cost of the project by diverting $\delta$ from the productive capital of the economy at date 0 . This is offset by investing $\delta \exp \left(r_{i} t\right)$ in the productive capital at date $t$. This implies that all cash flows are concentrated at date $t$. In expectation,

\footnotetext{
${ }^{2}$ See Gollier (2004, 2009a, 2009b).
} 
this net future value is positive if

$$
\epsilon \geq \delta \exp \left(R^{G}(t) t\right)
$$

where the time-dependent internal rate of return $R^{G}(t)$ satisfies the condition

$$
\exp \left(R^{G}(t) t\right)=\sum p_{i} \exp \left(r_{i} t\right)
$$

This can be rewritten as

$$
R^{G}(t)=\frac{1}{t} \ln \left(\sum_{i=1}^{n} p_{i} \exp \left(r_{i} t\right)\right) .
$$

It is not difficult to show that $R^{G}(t)$ defined by (7) has the properties

$$
R^{G}(0)=\sum_{i=1}^{n} p_{i} r_{i}, \frac{d R^{G}(t)}{d t}>0, \quad R^{G}(\infty)=\max \left\{r_{i}\right\}
$$

Unfortunately, Gollier did not at first provide a rigorous story to accompany his idea of using the internal rate of return defined by (7). Instead, like Weitzman, his argument was initially presented at the intuitive or heuristic level.

Aside from sharing the same initial condition $R^{W}(0)=R^{G}(0)=\sum p_{i} r_{i}$, the properties of $R^{G}(t)$ and $R^{W}(t)$ are diametrically opposed (except for the trivial situation in which there is but one sure future state of the world, in which case the two are criteria are identical). While $R^{W}(t)$ declines over time to the smallest value of $\left\{r_{i}\right\}$, on the contrary $R^{G}(t)$ increases over time to the largest value of $\left\{r_{i}\right\}$.

Both the Weitzman discount rate $R^{W}(t)$ and the Gollier discount rate $R^{G}(t)$ have a superficial plausibility, but with completely opposite conclusions and policy implications. If the correct discount rate is one of (2) or (7), then of necessity the other one must be wrong and will give the wrong answers to CBA questions. For want of a better name, this seeming paradox has been dubbed in the literature the "Weitzman-Gollier puzzle," and it has featured prominently in several papers about long-term discounting. ${ }^{3}$ How might a person resolve this distressing paradox by choosing between two such seemingly symmetric formulations, with each one having diametrically opposed implications for distant-future discounting? The answer can only come from a careful rigorous analysis, which follows in the next section.

\footnotetext{
${ }^{3}$ See, e.g., Buchholz and Schumacher (2008) and Freeman (2009).
} 


\section{Resolving the "Weitzman-Gollier Puzzle"}

As Gollier pointed out early on, and elucidated more carefully later, ${ }^{4}$ neither the formula for $R^{W}(t)$ given by (2) nor the formula for $R^{G}(t)$ given by (7) are likely correct as they stand when the evaluation of the safe investment is adjusted for the risk associated to its financing strategy. Indeed, both formulations contain a germ of truth that can be turned into a rigorous argument when expressed in units of marginal utility along an optimal consumption trajectory. Furthermore, and most importantly, the two rigorous formulations give the same discount rate (as a function of time), thereby resolving the "Weitzman-Gollier puzzle."

While several generalizations are possible, we focus here on the simplest case, the better to bring across the main points sharply. Our formal argument proceeds as follows. Break up time into a series of discrete periods $0,1,2, \ldots t, \ldots$ of unit length with corresponding consumptions $\mathbf{C}=\left(C_{0}, C_{1}, \ldots, C_{t}, \ldots\right)$. We postulate a very general utility function, in the spirit of Irving Fisher, of the form $V(\mathbf{C})$. We assume that $V$ is smoothly differentiable, is strictly concave, has positive first derivatives, and satisfies some kind of generalized Inada conditions that will guarantee unique interior solutions. Pure time preference is already built into this general utility function. A special case of $V(\mathbf{C})$ is the Ramsey-Koopmans form

$$
V(\mathbf{C})=\sum_{t=0}^{\infty} \exp (-\rho t) U\left(C_{t}\right),
$$

where $\rho>0, U^{\prime}(0)=\infty$ and $U^{\prime}(\infty)=0$. However, in some ways this special case (9) obscures rather than illuminates the act of seeing through to the core theoretical structure driving the model's results.

There is just one commodity serving as both consumption and investment. The notion of capital here is intended to be all-inclusive, including human capital, knowledge capital, and so forth. The underlying production function of this "generalized capital" is linear. In state of the world $i$ it is of the form

$$
K_{t+1}=\exp \left(r_{i}\right)\left[K_{t}-C_{t}\right]
$$

where $K_{t}$ is the capital stock at the beginning of period $t$. The linearity of the production possibilities frontier guarantees that the relevant interest rate in state $i$ will be $r_{i}$ - no matter what is the form of the general Fisherian utility function $V$.

It is critical in this model to understand the exact timing sequence concerning what information is available at what time, and when decisions are made. The base-case scenario is this. At time 0, the inherited capital stock is given as $K_{0}$ and the state of the world

\footnotetext{
${ }^{4}$ See Gollier (2009a, 2009b).
} 
is known. It is assumed that the deterministic problem of maximizing $V(\mathbf{C})$ subject to (10) has a unique interior bounded solution for all $i$. Denote the deterministic optimal consumption trajectory in state $i$ as $\mathbf{C}_{i}^{*}=\left(C_{0 i}^{*}, C_{1 i}^{*}, \ldots, C_{t i}^{*}, \ldots\right)$. From the linear production possibilities frontier (10), reducing consumption at date 0 by one unit in state $i$ would result in $\exp \left(r_{i} t\right)$ extra units available for increased consumption at time $t$ (without altering the rest of the optimal trajectory). Such a marginal change in the consumption plan should have no effect on intertemporal welfare $V$. Therefore, the optimal deterministic trajectory in state $i$ must satisfy the first-order condition

$$
\frac{\partial V\left(\mathbf{C}_{i}^{*}\right)}{\partial C_{0}}=\frac{\partial V\left(\mathbf{C}_{i}^{*}\right)}{\partial C_{t}} \exp \left(r_{i} t\right)
$$

for all $t$. This condition, which holds in all states $i=1, \ldots, n$, states that investors are indifferent about the financing structure of their projects.

Next suppose that an investment opportunity arises at time $t=0^{-}$, just before the "true" state of the world is revealed at time $t=0$. This safe investment opportunity expends marginal cost $\delta$ in order to yield a marginal benefit $\epsilon$ at future time $t$. Of course the representative agent wishes that the investment decision could be made with the precise information available at time $t=0$, just after the "true" state of the world is revealed and the relevant future marginal product of capital (from time $t=0$ to time $t=\infty$ ) is known with certainty. But the essence of the problem of doing CBA with an uncertain future discount rate is that the investment decision must be made at a time when it is known only that the uncertain future marginal product of capital will be $r_{i}$ with probability $p_{i}$.

By the envelope theorem and given the optimality condition (11), the possibility to reallocate the costs and benefits of the project over time has no effect on its impact on $V$, at the margin. This is the key to resolve the puzzle, as we demonstrate it below.

The investment project raises the expected utility of the representative agent if and only if

$$
\epsilon \sum_{i=1}^{n} p_{i} \frac{\partial V\left(\mathbf{C}_{i}^{*}\right)}{\partial C_{t}} \geq \delta \sum_{i=1}^{n} p_{i} \frac{\partial V\left(\mathbf{C}_{i}^{*}\right)}{\partial C_{0}} .
$$

Using the optimality condition (11), this can be rewritten in two equivalent ways. The "Weitzman approach" consists in eliminating $\partial V\left(\mathbf{C}_{i}^{*}\right) / \partial C_{t}$ from the above inequality. It yields

$$
\varepsilon \sum_{i=1}^{n} q_{i}^{W} \exp \left(-r_{i} t\right) \geq \delta \quad \text { with } \quad q_{i}^{W} \equiv \frac{p_{i} \frac{\partial V\left(\mathbf{C}_{i}^{*}\right)}{\partial C_{0}}}{\sum_{i=1}^{n} p_{i} \frac{\partial V\left(\mathbf{C}_{i}^{*}\right)}{\partial C_{0}}}
$$


This is equivalent to discount the future flow $\varepsilon$ at a rate $R_{*}^{W}(t)$ defined as follows:

$$
R_{*}^{W}(t)=-\frac{1}{t} \ln \left(\sum_{i=1}^{n} q_{i}^{W} \exp \left(-r_{i} t\right)\right)
$$

Alternatively, the "Gollier approach" consists in eliminating $\partial V\left(\mathbf{C}_{i}^{*}\right) / \partial C_{0}$ from (12) by using condition (11). It yields

$$
\varepsilon \geq \delta \sum_{i=1}^{n} q_{i}^{G}(t) \exp \left(r_{i} t\right) \quad \text { with } q_{i}^{G}(t) \equiv \frac{p_{i} \frac{\partial V\left(\mathbf{C}_{i}^{*}\right)}{\partial C_{t}}}{\sum_{i=1}^{n} p_{i} \frac{\partial V\left(\mathbf{C}_{i}^{*}\right)}{\partial C_{t}}} .
$$

This is equivalent to discount the future flow $\varepsilon$ at a rate $R_{*}^{G}(t)$ equaling

$$
R_{*}^{G}(t)=\frac{1}{t} \ln \left(\sum_{i=1}^{n} q_{i}^{G}(t) \exp \left(r_{i} t\right)\right) .
$$

Observe the close links between the definition of $\left(R_{*}^{W}(t), R_{*}^{G}(t)\right)$ in (14) and (16) and the definition of $\left(R^{W}(t), R^{G}(t)\right)$ in $(2)$ and $(7)$. They are equal up to a risk adjustment of probabilities. Weitzman converts all cash flows into consumption at time 0 and adjusts state-contingent net present values with units of marginal utility at time 0. Gollier converts all cash flows into consumption at time $t$ and adjusts net future values with units of marginal utility at time $t$. These risk adjustments are crucial because, although the properties of $R^{W}(t)$ and $R^{G}(t)$ are very heterogeneous, it is very easy to check from the optimality condition (11) that

$$
R_{*}^{W}(t)=R_{*}^{G}(t)
$$

for all $t$ ! This means that the adjustment of the valuation for risk resolves the "WeitzmanGollier puzzle". Let $R_{*}(t)$ denote this efficiently risk-adjusted discount rate.

It remains to explore the properties of this common risk-adjusted discount rate. Using the easier formulation $R_{*}=R_{*}^{W}$, it is not difficult to show that

$$
R_{*}(0)=\sum q_{i}^{W} r_{i}, \frac{d R_{*}(t)}{d t}<0, \quad R_{*}(\infty)=\min \left\{r_{i}\right\}
$$

so that qualitatively the properties of the efficient discount rate $R_{*}(t)$ resemble closely those of $R^{W}(t)$ recommended by Weitzman, with the only quantitative difference being the substitution of "Weitzman-adjusted probabilities" $\left\{q_{i}^{W}\right\}$ for the unadjusted probabilities $\left\{p_{i}\right\}$. It is good to provide an intuition to the result that the term structure of the socially efficient discount rate be decreasing. In this model in which shocks on capital productivity are permanent, risk on consumption growth are also permanent, as seen from equation (11). This 
implies that risks are magnified by time, compared to the more standard Brownian motion in which it is known that the term structure of discount rate is flat. What are the consequences of this magnification of long term risk on the discount rate for long maturities? Intuitively, future risk should induce prudent consumers to sacrifice more for this future. This is the Keynesian notion of precautionary saving. This is translated into using a smaller discount rate.

Let us illustrate this result by considering the special case of the Ramsey-Koopmans specification (9) for $V$. Let us in particular consider the case of the logarithmic utility function $U(C)=\ln C$. In that case, one can rewrite the optimality condition (11) as $C_{i t}=$ $C_{i 0} \exp \left(r_{i}-\rho\right) t$. Rewriting the intertemporal budget constraint (10) as $K_{0}=\Sigma_{t} C_{i t} \exp \left(-r_{i} t\right)$ and eliminating $C_{i t}$ yields that

$$
C_{i 0}=K_{0}(1-\exp (-\rho))
$$

for all $i=1, \ldots, n$. Observe that the initial consumption is risk free in this special case. As is well-known, consumption is not affected by a change in the interest rate in the logarithmic case. It implies that one is neutral to the small risk affecting the net present value of the project at date 0 , which implies in turn that the Weitzman rule $R^{W}(t)$ given in equation (2) is correct in that case. This can be seen by observing in (13) that $q_{i}^{W}=p_{i}$ for all $i$, so that $R_{*}^{W}(t)=R_{*}(t)=R^{W}(t)$ ! In this case, Weitzman's rule was qualitatively and quantitatively correct.

\section{Concluding Remarks}

We will not bother to go through all of the many caveats that should attach to the results of this paper. Nor shall we discuss possible extensions to more complicated and realistic situations.

The bottom-line message that we wish for readers to take away from this paper is the following. When future discount rates are uncertain but have a permanent component, then the "effective" discount rate must decline over time toward its lowest possible value. Empirically, this important feature can have significant ramifications for climate-change CBA - by weighting the distant future much more heavily than is done by standard exponential discounting at a constant rate. ${ }^{5}$

\footnotetext{
${ }^{5}$ See, e.g., Newell and Pizer (2003), or Groom, Koundouri, Panopoulou and Pantelidis (2007).
} 


\section{References}

[1] Buchholz, W., and J. Schumacher (2008). "Discounting the long distant future: A simple explanation for the Weitzman-Gollier puzzle. Working paper: University of Regensburg.

[2] Dasgupta, P. (2008). "Discounting climate change. Journal of Risk and Uncertainty, 37: 141-169.

[3] Freeman, M. C. (2009) "Yes, We should Discount the Far-Distant Future at Its Lowest Possible Rate: A Resolution of the Weitzman-Gollier Puzzle." Economics E-journal Discussion Paper 2009-42.

[4] Gollier, C. (2004) "Maximizing the expected net future value as an alternative strategy to gamma discounting." Finance Research Letters 1 (2): 85-89.

[5] Gollier, C. (2009a). "Should we discount the far-distant future at its lowest possible rate?." Economics: the Open-Access, Open-Assessment E-Journal, vol. 3: 2009-25.

[6] Gollier, C. (2009b). "Expected net present value, expected net future value, and the Ramsey rule." CESifo working paper \#2643.

[7] Groom, B., P. Koundouri, E. Panopoulou and T. Pantelidis (2007). "Discounting the distant future: how much does model selection affect the certainty equivalent rate?". Journal of Applied Econometrics. 22 (3): 641-656.

[8] Hepburn, C. and B. Groom (2007). "Gamma discounting and expected net future value." Journal of Environmental Economics and Management. 53 (1): 99-109.

[9] Newell, R. and W. A. Pizer (2003). "Discounting the distant future: how much do uncertain rates increase valuations?". Journal of Environmental Economics and Management 46 (1): $52-71$.

[10] Newell, R. G. and W. A. Pizer (2004). "Uncertain discount rates in climate policy analysis." Energy Policy 32 (4): 519-529.

[11] Pazner, E. A. and A. Razin (1975). "On expected present value vs. expected future value. Journal of Finance 30 (3): 875-877.

[12] Weitzman, M. L. (1998) "Why the Far-Distant Future Should Be Discounted at Its Lowest Possible Rate." Journal of Environmental Economics and Management 36 (3): 201-208. 
[13] Weitzman, Martin L. (2001). "Gamma Discounting." American Economic Review, 2001 (March), 91 (1): 260-271. 